\section{Avaliação antropométrica do estado nutricional da gestante: visão retrospectiva e prospectiva}

\section{Anthropometric assessment of the pregnant nutrition status: retrospective and prospective view}

Kênia Siqueira Coelho 1

Ariani Impieri de Souza 2

Malaquias Batista Filho 3

${ }^{1}$ Hospital Sarah Kubitshek. Salvador, Bahia.

2 Núcleo de Pesquisa. Instituto Materno Infantil de Pernambuco, IMIP.

3 Departamento de Nutrição. Centro de Ciências da Saúde da

Universidade Federal de Pernambuco. Instituto Materno Infantil de Pernambuco, IMIP.

Núcleo de Pesquisa. Instituto Materno Infantil de Pernambuco,

IMIP. Rua dos Coelhos, 300. Recife, Pernambuco, Brasil. CEP

50.750-550. Tel/Fax: (81) 3413.2264

\begin{abstract}
The authors analyzed theoretical foundations, criteria and technical models used for the last 40 years in the anthropometric assessment of nutrition status during pregnancy. They propose that new studies aiming at the methodological solution of this problem shoud not include" birthweight" as a prevailing reference in evaluating this method, considering the requirement aprioristic, not holding much empirical consistence.

Key words Anthropometry, Nutritional assessment, Pregnancy
\end{abstract}

\section{Resumo}

Os autores analisam os fundamentos teóricos, critérios e modelos técnicos utilizados nos últimos 40 anos na avaliação antropométrica do estado nutricional durante a gravidez. Propõem que novos estudos objetivando uma solução metodológica para o problema da avaliação somatométrica da gestante devem ser desvinculados da condição "peso ao nascer" como referencial dominante na avaliação do método, considerando este requisito como um apriorismo sem consistência com os resultados observados.

Palavras-chave Antropometria, Avaliação nutricional, Gestantes 
No processo gravídico, o estado de nutrição apresenta uma dupla relevância, sob o ponto de vista clínico e epidemiológico, desde que interessa à mãe, por representar um período de reconhecida vulnerabilidade biológica, e ao concepto, extremamente dependente do organismo materno para seu crescimento e desenvolvimento. 1 No ciclo da vida humana, provavelmente, a gravidez representa o processo ou o momento fisiológico mais crucial, face às demandas e circunstâncias em que se desenvolve.2,3

Apesar do reconhecimento consensual desta importância1,4,5 existem ainda consideráveis questionamentos sobre os métodos utilizados e até mesmo sobre os fundamentos conceituais que devem direcionar os estudos e pesquisas sobre avaliação do estado nutricional da gestante. Trata-se, assim, de uma limitação que dificulta, enormemente, a elaboração de juízos clínicos e dos procedimentos que deles devem decorrer.

A avaliação da idade gestacional, o ganho de peso gravídico, o efeito de confusão que resulta da retenção fisiológica ou patológica de líquidos no leito vascular (hemodiluição) ou no espaço intersticial, produzindo ou não edema clinicamente evidente, as alterações na composição corporal que ocorrem no período de vida reprodutiva representam, entre outros fatores, variáveis que dificultam a aplicação universal de parâmetros antropométricos no curso da gestação.2,6 Como proceder, em termos normativos, para compatibilizar estas diversas interferências na determinação e interpretação do peso gestacional?

Por outro lado, a tendência praticamente generalizada de condicionar a definição do estado nutricional materno ao peso do recém-nascido estabelece outro campo de complicações conceituais e técnicas, devido à grande variedade de fatores que atuam na determinação do peso ao nascer.7-9 Desse modo, duas vertentes de problemas se conjugam: de um lado, a multiplicidade de fatores que interferem sobre o peso gravídico e, de outra parte, a variedade de condições que determinam o peso ao nascer, 10 incluindo características prévias à gravidez, como idade, paridade, dimensões corporais e características da evolução da gestação, como ganho de peso, tabagismo, presença de doenças intercorrentes e recebimento de assistência pré-natal.11-13

Várias tentativas têm sido realizadas no sentido de se encontrar uma solução científica e operacionalmente satisfatória para avaliação do estado nutricional da gestante, de forma a atender aos conceitos e critérios em que a questão tem sido colocada. Neste sentido, os métodos antropométricos, de importância já reconhecida como instrumento de medi- da da condição nutricional, têm centralizado a atenção e os esforços dos pesquisadores, sendo consideráveis os progressos obtidos nas últimas quatro décadas. ${ }^{14-16}$

Em 1961, um grupo de especialistas do Instituto de Nutrição de Centro-América e Panamá (INCAP) 14 construiu um modelo gráfico onde eram consideradas as diferenças de estatura materna e o aumento provável de peso em função da idade gestacional que poderia ser aceito como limite superior e inferior de normalidade. Por meio desse modelo, que tinha como fundamento uma linha de base para o peso pré-gravídico, poderia ser estabelecido um "programa" de evolução ponderal a ser acompanhado durante os exames pré-natais, classificando-se as posições das gestantes no gráfico como "peso insuficiente", "peso normal" e "sobrepeso". Este método foi extensivamente utilizado em países centro-americanos, difundindo-se também em muitos serviços de saúde de algumas nações da América do Sul. O gráfico mantinha uma limitação crucial, pois não considerava as implicações do estado nutricional prévio.

Aceitava-se o mesmo padrão de aumento do peso gravídico em gestantes obesas ou com "déficit" ponderal no início da gravidez ou no momento do exame pré-natal, o que representava, conceitualmente, uma evidente impropriedade, deixando de considerar, justamente, o risco de uma desnutrição materna que pode preceder o desenvolvimento do processo gravídico. 15,17

Considerando esta limitação, um grupo de especialistas, em São Paulo, ${ }^{14}$ propôs e testou uma modificação no modelo gráfico do INCAP, definindo "linhas de base" que levavam em conta a relação peso/altura e seu campo normal de variação, de forma a controlar as situações inicias de sobrepeso ou de baixo peso para a estatura materna. O modelo então resultante foi testado a partir de uma casuística de cerca de 2.000 gestantes acompanhadas da gravidez até o parto, na cidade de São Paulo. Tendo como um dos objetivos a indicação do risco de baixo peso ao nascer, o método foi posteriormente normatizado, 17 passando a ser adotado em vários serviços de saúde do país. Tratava-se, no entanto, de um modelo conceitual definido por consenso e com pontos de corte estabelecidos por convenção, sem a necessária validação interna, desde que a experiência de Siqueira et al.,15 não implicou na modificação das linhas demarcatórias estabelecidas em bases essencialmente conceituais, como um gráfico tentativo.

Um novo modelo gráfico foi proposto posteriormente por Lechtig e Klein, 18 apoiado na distribuição centilar do aumento de peso gravídico e sua capacidade de discriminar o risco de baixo peso ao nascer. 
Baseado em experiências com gestantes da América Central, com características étnicas ou fenotípicas bem singulares, o método se ressentia de evidências empíricas que recomendassem seu uso generalizado (validação externa).

Em 1985, partindo de várias observações, Rosso 16 propôs, para fins de validação, um estudo multicêntrico (Estados Unidos, Chile e Brasil) testando um modelo gráfico com várias linhas de adequação do peso gestacional. A curva citada baseia-se na constatação de que, ao final da gestação, o ganho de peso de $20 \%$ em relação ao peso ideal para a estatura no início da gravidez assegura o máximo crescimento do feto. A consistência dos primeiros resultados fez com que, no Brasil, fosse adotada e recomendada, em caráter preliminar, a chamada Curva Ponderal da Gestante (Curva de Rosso) como critério de avaliação do peso gravídico. Apesar do caráter preliminar e, portanto, transitório, até hoje esse modelo continua sendo aplicado nos serviços públicos de saúde do país. 19

O uso extensivo da chamada "Curva de Rosso" no Brasil possibilita a esta altura, uma observação generalizada, ou seja, a discordância, em termos epidemiológicos, entre a situação nutricional das gestantes atendidas em serviços de saúde e o estado de nutrição de mulheres no período reprodutivo. Em termos concretos: enquanto a avaliação nutricional de mulheres no período gravídico evidenciaria um problema de elevadíssima magnitude (35\% a $45 \%$ e até mais de desnutrição), os estudos de campo aplicados em mulheres adultas em idade reprodutiva evidenciariam, em escala nacional, que não mais de $6 \%$ seriam considerados em situação de deficiência calórico-protéica (DCP) pela aplicação do Índice de Massa Corporal (IMC). 20 Assim, a comparação de dados ecológicos resultaria na demonstração de que a desnutrição apresentaria um risco relativo cinco a sete vezes maior na gestante, quando referenciada em relação ao mesmo contingente de idade da população de mulheres adultas. Dado de realidade ou artefato de um viés metodológico, tipo "dois pesos e duas medidas"?

Em Recife, um grupo de pesquisadores, estudando a Circunferência Braquial e a Curva de Rosso, observou que existem consideráveis diferenças entre os resultados do estado nutricional segundo o método de Rosso e os dados do perímetro braquial materno, como indicadores preditivos do peso ao nascer, no ponto de corte de $23,5 \mathrm{~cm}$, universalmente recomendado.21,22 Trata-se de um método promissor, desde que a circunferência braquial é uma medida praticamente independente da idade gestacional. 23 Restava no entanto, uma grande dúvida: a aceitação da curva de Rosso como método referencial (gold standard). Assumindo, por convenção, que a curva de Rosso representaria o padrão ouro de classificação antropométrica, determinou-se que, no limite de $25 \mathrm{~cm}$, os resultados das duas classificações se tornavam estatisticamente indiferenciados.

Estudos de Atalah et al.,24,25 baseados no Sistema de Informação Perinatal do Ministério da Saúde do Chile, também demonstraram que existe uma baixa concordância entre o diagnóstico nutricional da gestante obtido com as normas atuais (Curva de Rosso) e outros critérios de classificação nutricional. Confirmava-se mais uma vez, a inconsistência dos resultados face à situação epidemiológica, prevalecendo a observação de que o método de Rosso resultava numa ocorrência hiperestimada de déficit ponderal no transcurso da gestação, que no Chile, país onde menos de 5\% das crianças nascem com baixo peso, 25 chegava a $50 \%$.

A World Health Organization (WHO) ${ }^{1}$ através de seu comitê de especialistas em Nutrição, considera que o peso gravídico não deve ser analisado apenas em função do objetivo de predição e monitorização do baixo peso do recém-nascido. Seria uma concepção reducionista, na medida em que, mesmo representando, em determinados contextos socioeconômicos e epidemiológicos, o "handicap" mais relevante em relação à saúde perinatal, não deve ser elevada à condição de objetivo hegemônico e, menos ainda, exclusivo. São vários os riscos para a mãe e para o concepto que derivam do peso de ambos no decurso e desenlace da gravidez, de tal forma que a concepção e a validação de um método de avaliação do estado nutricional devem estar abertas para um espectro variado e às vezes conflitivo de riscos opostos.

Partindo dessas observações, Atalah et al.,25 desenvolveram um instrumento de avaliação nutricional da gestante baseado no IMC, objetivando, simultaneamente, minimizar os riscos nutricionais para a mãe e para o feto. De forma muito pertinente os autores levaram em consideração três fatos muito significativos, em termos de nutrição e saúde coletiva. O primeiro, se referia à redução considerável, em escala nacional, da prevalência da desnutrição materna, que teria declinado em $35 \%$, no Chile, dentro de 10 anos. O segundo, relacionado com o anterior, se configurava no fato de que, no mesmo período, a ocorrência de obesidade mais do que duplicou, elevando-se em $110 \%$, enquanto simultaneamente (e este representa o terceiro aspecto, em termos de interesse epidemiológico pelo estado nutricional do binômio mãe/concepto) a incidência de baixo peso ao nascer (BPN) caiu para valores abaixo de $5 \%$, 
caracterizando, portanto, uma situação semelhante à encontrada em países com padrões satisfatórios de saúde e nutrição. 25,26

$\mathrm{O}$ confronto desses dados, além de expor à reflexão clínica resultados que não podem ser omitidos (baixa ocorrência de desnutrição materna e de baixo peso ao nascer, além do crescimento surpreendente das taxas de obesidade), induziu à consideração dos formuladores de programas e de normas técnicas de procedimento, a necessidade de revisar a questão da classificação do estado nutricional da gestante e os próprios fundamentos desta avaliação, diante das evidências de que a busca e tratamento do baixo peso gestacional como meio de prevenir o baixo peso ao nascer, já não deveria monopolizar os cuidados nutricionais prestados nos serviços de saúde.

Isto incluiria o interesse de considerar, perceber e monitorizar os riscos bilaterais (mãe e feto) que podem resultar da obesidade no transcurso da gravidez, conferindo, portanto, ao método de avaliação antropométrica uma abrangência de aplicações bem mais universal que os modelos precedentes.

Referenciados nesses objetivos, o método original de Atalah et al., ${ }^{25}$ consiste, na realidade, na aplicação convencional do IMC ajustado para a idade gestacional, de tal modo que o fundamento conceitual do método tem como suporte um indicador já bem consolidado por um grande número de trabalhos validativos.27,28 Assim, o caráter tentativo dos métodos antropométricos de avaliação assume uma posição mais ponderada e portanto, mais consistente e convincente.

A partir de uma pequena casuística, o Centro
Latinoamericano de Perinatologia propôs um modelo tentativo de avaliação antropométrica do estado nutricional da gestante utilizando o aumento de peso a partir de 12 semanas de gravidez, consistindo em quatro curvas correspondentes aos percentis 10, 25, 50 e 90.29 Este modelo foi incorporado no manual técnico de "Assistência pré-natal" do Ministério da Saúde do Brasil. 30 resultando em controvérsias da parte dos estudiosos do problema. Além do pequeno número de observações o chamado método de Fescina29 fundamenta-se em relações corporais de mulheres no período reprodutivo que compunham tabelas de referência peso/idade na metade do século passado, quando a antropometria nutricional ainda não incorporava normas e padrões hoje considerados.

É nesta perspectiva que se coloca a presente proposta de rever o tema, admitindo-se que a partir de novos pressupostos e conceitos se pode alcançar um método mais satisfatório de avaliação do estado nutricional da gestante, eliminando em grande parte os casos de falsos positivos, que, como já sabemos, superestimam o problema da desnutrição pelo método de Rosso. 16

Isto implica, entre outras coisas, em não considerar sistematicamente que o peso ao nascer seja o critério definitivo de validação dos métodos antropométricos de avaliação do estado nutricional da gestante. Esta posição, que se tornou convencional e quase clássica, pode constituir um apriorismo conceitual e metodológico que termina impedindo ou dificultando a busca de novos caminhos para resolver um velho problema. 


\section{Referências}

1. WHO (World Health Organization). Physical status: the use and interpretation of anthropometry: report of a WHO Expert Committee. Geneva: WHO; 1995. (Serie 854).

2. Guyton AC. Tratado de fisiologia médica. 7. ed. Rio de Janeiro: Guanabara Koogan; 1989.

3. Krasovek K, Anderson MA. Maternal nutrition and pregnancy outcomes: antropometric assessment. Washington, DC: WHO (World Health Organization); 1991. (Scientific Publication 529).

4. Kalkwark HJ. Maternal weight gain during pregnancy and risk of preterm delivery: effects on neonatal mortality and public health impact. In: WHO (World Health Organization). Physical status: the use and interpretation of anthropometry: report of a WHO Expert Committee. Geneva: WHO; 1995. p. 37-120. (Serie 854).

5. Kramer MS. Intrauterine growth and gestational duration determinants. Pediatrics 1987; 80: 502-11.

6. Rezende J. Obstretícia. 7. ed. Rio de Janeiro: Guanabara Koogan; 1995.

7. Savitz DA, Blackmore CA, Thorp JM. Epidemiologic characteristics of preterm delivery: etiologic heterogenity. Am J Obstet Gynecol 1991; 164: 467-71

8. Lawrence M, McKillop FM, Durnin JVG. Women who gain more fat during pregnancy may not have bigger babies: implications for recomended weight gain during pregnancy. Br J Obstet Gynecol 1991; 98: 254-9.

9. Haidar FH, Oliveira UF, Nascimento LFC. Escolaridade materna: correlação com os indicadores obstétricos. Cad Saúde Pública 2001; 17: 1025-9.

10. Scott KE, Usher R. Fetal malnutrition: its incidence, causes and effects. Am J Obstet Gynecol 1966; 94: 951-63

11. Benício MHD'A. Análise multivariada de fatores de risco para o baixo peso ao nascer de nascidos vivos do município de São Paulo, SP (Brasil). Rev Saúde Pública 1985; 19: $311-20$

12. Rosso P. Nutrition and maternal fetal exchange. Am J Clin Nutr 1981; 34: 744-55

13. Van der Berg BJ. Maternal variables affeting fetal growth. Am J Clin Nutr 1981; 34: 722-6.

14. INCAP (Instituto de Nutrición de Centro-América Panamá). Evaluación del peso de la embarazada. Guatemala: INCAP; 1961; (Nutrición en Salud Pública, NSP, 3).

15. Siqueira AAF, Mattos ILB, Batista Filho M. Aplicação de uma curva de ganho de peso para a gestante. Rev Saúde Pública 1977: 11: 288-93.
16. Rosso P. A new chart to monitor weight gain during pregnancy. Am J Clin Nutr 1985; 41: 644-52.

17. Batista Filho M, Monteiro EAA. Operacionalização da curva ponderal da gestante. Rev Aliment Nutr 1984; 16: 44

18. Lechtig A, Klein RE. Guia para interpretar la ganância de peso durante el embarazo como indicador de riesgo de bajo peso al nacer. Bol Ofic Sanit Panam 1980; 89: 48995

19. Ministério da Saúde. Assistência pré-natal: normas e manuais técnicos. 21. ed. Brasília, DF: Ministério da Saúde; 1988

20. Monteiro CA, Mondini L, Souza ALM, Popkin BM. Da desnutrição para a obesidade: a transição nutricional no Brasil. In: Monteiro CA. Velhos e novos males da saúde no Brasil. São Paulo: Hucitec; 1995.

21. Barros LMF. A circunferência braquial e a curva de ganho ponderal como métodos de avaliação do estado nutricional da gestante [dissertação mestrado]. Recife: Departamento de Nutrição, Centro de Ciências da Saúde da Universidade Federal de Pernambuco; 1990.

22. Batista Filho M, Barros LF, Nacul LC. O perímetro braquial como método de avaliação do estado nutricional das gestantes. Revista do IMIP 1993; 7: 12-6.

23. Bishop CW, Pitchey SJ. Estimation of the mid-upper arm circunference meassurement error. J Am Dietics Assoc 1987; 87: 469-73.

24. Atalah SE, Castillo CL, Gomez C. Desnutrición em la embarazada: um problema sobredimensionado? Rev Med Chile 1995; 123: 1531-8

25. Atalah SE, Castillo CL, Castro RS. Propuesta de um nuevo estandar de evaluacion nutricional em embarazadas. Rev Med Chile 1997; 125: 1429-36.

26. UNICEF (Fundo das Nações Unidas para a Infância). Situação mundial da Infância: 2000. Brasília, DF: UNICEF 2000

27. The functional significance of low body mass index (BMI): proceedings of na IDECG workshop; 1992 Nov 4-6; Rome, Italy. Eur J Clin Nutr 1992; 48 Suppl 3: S1-202.

28. Anjos LA. Índice de massa corporal como indicador do estado nutricional de adultos: uma revisão da literatura. Rev Saúde Pública 1992; 26: 431-6.

29. Fescina R. Nutricion materna y resultados perinatales. Clin Perinatol Argentina 1996/1997; 4: 77-98

30. Ministério da Saúde. Assistência pré-natal: manual técnico. Brasília, DF: Ministério da Saúde; 2000. 